\title{
TRA LA «MAPPA PER I SETTE MARI» ED IL «LIBRO DELLA MARINA»DI PIRI REIS
}

Nel 1988-89 sono stati pubblicati, a cura del Ministero della Cultura e Turismo della Repubblica Turca e della «Historical Research Foundation - Istanbul Research Center", in edizione fototipica con trascrizione del testo ottomano e traduzione in turco ed in inglese, il primo ed il secondo dei quattro preventivati volumi editoriali del Kitab-i Babriye di Piri Reis ', il grande uomo di mare, geografo e cartografo turco vissuto tra il 1465-70, circa, ed il $1554^{2}$. Nato a Gelibolu (Gallipoli), la bella città costiera sul Mar di Marmara ${ }^{3}$, era nipote del celebre ammiraglio Kemâl Reis, e si chiamava Muhiddin Piri. Di lui si può dire ciò che Ibni Kemal, lo storico turco, scrive dei ragazzi di Gelibolu: "Crescono nell'acqua come alligatori. Loro culle sono le barche. Sono indotti al sonno dalla ninna-nanna del mare e delle navi, di giorno e di notte" ${ }^{4}$.

Figlio di Haci Mehmet, entrò, più o meno che dodicenne, negli equipaggi delle navi dello zio, che era "Azaphar Reisi»" al servizio del bey di Eg̈riboz e con il quale trascorse ininterrottamente tredici o quattordici anni, prendendo parte alle sue attività corsare. Possiamo seguirlo in questo periodo della sua vita grazie a quanto egli ci dice nel suo libro, dove ricorda, in uno stile quanto mai piacevole, l'esperienza nei luoghi visitati con Kemâl, insieme con notizie sugli avvenimenti storici dell'epoca. Le sue informazioni sulle isole e sulle coste dell'Egeo, come pure le sue mappe, sono molto interessanti. Vediamo, ad esempio, che cosa egli scrive sulle due Focee: le due località contigue sulla costa anatolica, ricche di ottime miniere di allume, prima feudo degli Zaccaria di Genova e poi dominio genovese dal 1346, passate in mano turca nel $1455^{\circ}$ :

«Eski Foça [Focea Vecchia] fu costruita originariamente da mercanti veneziani,

1 Nel presente lavoro abbiamo reso la i turca senza punto con i.

2 Ben poco si conosce della vita di Piri Reis; per di più molti dati sono soggetti a seri dubbi. Cfr., ad ogni modo, S. SOUCEK, $\dot{A}$ propos du liure d'instructions nautiques de Piri Reis, in «Revue des Études Islamiquesw, xLI.2, 1973, pp. 241-255, con la bibliografia ivi citata; $A$. AfETINAN, Lifo and works of Piri Reis. The oldest map of America, Ankara, 1975 (bibliografia a pp. 73-78).

3 Gallipoli fu il centro da cui cominciarono a partire le prime spedizioni e scorrerie ottomane nel Mediterraneo. Cfr. F. Babinger, Mebmed der Eroberer und seime Zeit, München, 1953, traduz. italiana di Eveluna POLAcco, Maometso il Conquistatore, Torino, 1957.

4 A. Afetinan, Lifo and Works cit., p. 9.

"L' «Azaplar Reisi» è il comandante di un corpo di marinai.

6 PH.P.ARGENTI, The occupation of Chios by tbe Genovese and tbeir administration ad tho island (1346-1566), Cambridge, 1958, vol. I; Geo Pistarino, Cbio dei Genovesi, in «Studi medievaliw, 3.' serie, X.1, 1969, pp. 3-68; Id.. Genovesi d'Oriente, Genova, 1990, pp. 243-280. 
mentre mercanti genovesi ebbero Yeni Foça [Focea Nuova]. E'ciò che ora comunemente si sa su questi due castelli. In tempi antichi, quando le coste dell'Anatolia erano ancora nelle mani degl'infedeli, molti mercanti erano usi venirvi da Venezia. Ricolmarono di doni il signore dell'Anatolia e gli chiesero un luogo sul quale potessero costruire un castello, assicurando quel sovrano che egli sarebbe stato garantito della rendita del castello e dichiarando che anche i mercanti ne avrebbero beneficiato. Essendo state approvate queste richieste, essi fondarono il castello di Eski Foça. Poiché Eski Foça divenne un luogo invidiabile, anche i mercanti genovesi avanzarono richiesta per un porto, al quale essi potessero portare le loro stoffe ed i loro beni. Così fondarono il castello di Yeni Foça: dopo di che qui non mancarono più mercanti genovesi. Persino al mio tempo io ho visto mercanti di Genova nella residenza di Yeni Foça. Oggi tuttavia se ne sono andati tutti: nessuno di loro vi rimane" '.

E' una notizia preziosa, perché risulta di qui evidente che, pure dopo la conquista turca del 1455, i mercanti genovesi frequentavano Focea, ancora all'epoca della giovinezza di Piri Reis, cioè intorno al 1477-82, mentre più non ce n'erano quando Piri scrisse il suo libro, cioè nel 1521. Ricordiamo che nel 147401475 anche Cristoforo Colombo navigò nell' Egeo, fu a Chio, di fronte alla costa turca di Focea, $\mathrm{e}$ dall'isola portò con sé, indimenticabile, il ricordo del profumo del mastice ${ }^{8}$.

Passiamo sulla parte opposta del quadro euro-mediterraneo. Nel 1486-87 il regno moresco di Granada, dilaniato dalle lotte intestine tra Boabdil e Zagal e stretto dagli attacchi di re Ferdinando di Castiglia, che nel 1486 occupò la munitissima piazzaforte di Loja e nell'anno successivo conquistò Velez-Malaga e Malaga, inviò richieste di soccorso alla Tunisia, all'Egitto ed al governo ottomano. In quell'inverno giunse ad Istanbul, alla corte di Bayazid II, un inviato dell'emiro granadino, Abu Abd - Allah el Zagal, con una supplica in versi, dovuta alla penna del poeta Abu'l - Beqa Salih ben Sherif, in cui si lamentavano «le sofferenze dei Musulmani, la caduta dell'Islam nella Spagna, la prossima cacciata degli Arabi dall'Andalusia, dopo che per settecento anni essi l'avevano posseduta».

Kemâl Reis, che si era dato ad attività corsara, prese il mare dalla base di Gelibolu nel 1487; raggiunse Gerba, Malta, la Sicilia, la Sardegna, la Corsica. Insidiò le coste dell'Italia meridionale, attaccò le Baleari e le coste aragonesi, bombardò ed incendiò Malaga, caduta in potere della Castiglia. Il Sultano non si era voluto compromettere direttamente, sicché le operazioni dell' ammiraglio parvero o furono fatte apparire come azioni di pirateria, anche se Kemâl Reis ottenne aiuto e tutela presso i principati islamici del Nord-Africa. Furono sei-sette anni di guerra marittima, dal 1487 al 1493, durante i quali Piri restò accanto allo zio, combattendo sia contro le forze cristiane sia contro altri pirati, e trascorrendo gl'inverni in porti amici, a Tunisi, a Bona, ad Algeri '.

7 Ediz. del Kitab-i Babriye di Piri Reis, a cura del Ministero della Cultura e del Turismo della Repubblica turca, vol. I, Istanbul, 1988, p. 325.

8 P.E. Taviani, Cristoforo Colombo: la genesi della grande scoperta, ediz. economica, Novera, 1982, cap. VIII.

9 A. Masala, La prima spedizione ottomana in Spagna (1487), in "Medioevo. Saggi e rassegne», 8, 1983, pp. 111-118; ID., La prima spedizione ottomana sulle coste della Spagna, in «La presenza italiana in Andalusia nel basso medioevo. Atti del Secondo Convegno, Roma, 22-27 maggio 1984", a cura di A. Boscolo e B. TORRES, Milano, 
Ecco la sua descrizione della Corsica genovese: "Sovrasta quest'isola un'alta montagna, che corre da nord a sud. Io ho contato 25 picchi montuosi nella sua parte orientale. Sembrano proprio i denti d'una sega. Ciascuno di questi picchi è coperto di neve durante tutto l'anno». Gli abitanti: "L'isola di Corsica era un dominio dei Genovesi, ma più tardi, quando i Francesi conquistarono Genova, tra gli altri anche quest'isola passò alla Francia» ${ }^{10}$. Piri è dunque bene informato dell'instaurazione del dominio francese a Genova con Luigi XII.

Vagando per il Mediterraneo, Piri Reis vide molti paesi: di ogni porto ove approdavano studiava le qualità e le proprietà, schizzandone una cartina: carte ed esperienze che raccolse poi nel libro Kitab- $i$ Babriyxe ( Libro della marina»). Visitò le città costiere della Spagna e dellÁlgeria: per sei anni egli e Kemâl Reis trasportarono musulmani spagnoli in Africa Settentrionale, cosa non facile e che li portò a sanguinose battaglie.

Rientrato in patria Kemâl Reis, Piri passò con lui al servizio regolare nella flotta ottomana nel 1495. Nel 1498 partì con lo zio per la guerra contro Venezia. Prese parte alle vittoriose battaglie di Lepanto e di Modone. Nominato ammiraglio, come pure lo zio, intervenne in scontri navali nel 1499-1502; fu comandante di gruppi di vascelli; svolse un servizio in azioni di guerra contro i veneziani nel 1500- 1502 . Quando Kemâl Reis morì, nel 1501-1502, Piri lo ricordò con parole commosse: «Molti se ne vanno, convinti di ritornare, ed alcuni non tornano; quelli che non tornano sono coloro i quali poco sanno di dove stanno andando. Sebbene la sua fama fosse un tempo dichiarata da tutti, ora persino il suo nome è perduto e scomparso. Questo mondo è tutto vanità: è destino di ogni uomo vivere o morire. Mentre egli era al servizio di Bayezid, Han di Azrael [l'Angelo della morte] se lo è preso con sé. Possa Iddio donare la pace a quanti ricordano il defunto Kemâl Reis con una preghiera! La vita di Kemâl Reis è giunta alla fine. Quando egli è andato nell'al di là, noi ci siamo sentiti più soli in questo mondo. Ha servito il Sultano per diciassette anni completi ed è morto nel 907 [1501-1502]. La stessa fine attende senza dubbio anche noi, e ciò che abbiamo detto cambierà" ". Dopo la morte dello zio Piri lasciò gli spazi dei mari aperti, si mise al lavoro, a Gallipoli, intorno alla sua prima mappa; raccolse le note per il suo libro.

Una delle principali caratteristiche di Piri è rappresentata dal fatto che, insieme ai suoi doveri militari, egli continuò, senza sosta, la sua opera di cartografo ed i suoi studi geografici, in guerra ed in pace. Nel 1513 redasse la sua prima carta, che fu poi presentata al sultano Selim I (1512-1520). Prese parte alla campagna navale contro Alessandria nel 1516-17: dopo la conquista del porto e la cattura delle navi in esso presenti, Piri si recò al Cairo e disegnò una mappa del delta del Nilo. L'anno seguente, quando il Sultano venne ad Alessandria con la flotta, Piri fu introdotto alla sua presenza e gli fece omaggio della sua carta. Nel 1521 ridusse a libro, in una prima stesura, le sue note di viaggio.

Durante il regno di Solimano il Magnifico (1520-1566), che fu un susseguirsi

1986, pp. 169-176; L.BALleTto, Tra Istambul ed il regno moresco di Granada sulla fini del Quattorocento, in *Atti del IV Convegno internasionale di Studi Colombiani» Genova, 1987, pp. 24-43.

10 A. Afetinan, Life and Works cit., pp. 11-12.

11 Ediz. del Kitab-i Babriye cit. I, p. 67. 
di belle vittorie, Piri ebbe un comando nella flotta ottomana, inviata nel 1522 contro Rodi, che fu tolta ai Cavalieri. In occasione di questa impresa, egli conobbe Ibrahim Pascià, che tanta parte ebbe nell'incoraggiarlo a ridurre a libro, in una seconda stesura, le sue annotazioni, per presentare il lavoro al Sultano. Il che avvenne nel 1526-27, con accoglienza favorevole. Fino a questa data noi possiamo seguire la biografia dell'Autore appunto grazie alla sua opera, che Piri così conclude: "Abbiamo esaudito il nostro desiderio e scritto questa strofa».

Frattanto i suoi titoli diventavano sempre più importanti. Fu insignito di quello di Ammiraglio del Mare di Oman e del Mar Rosso, mentre i suoi viaggi si erano estesi dal Mediterraneo al Mar Rosso, al Golfo Persico, all'Oceano Indiano: ed è il settore a cui si estesero le sue competenze militari, grazie anche alla conoscenza ch'egli ne aveva acquisita.

In seguito alle crescenti attività portoghesi nell'Oceano Indiano, nel Golfo Persico e nella parte meridionale del Mar Rosso, l'Impero ottomano creò infatti un comando militare, denominato "Hind Beylerbeyligi» o «Hind Kaptanliği ${ }^{12}$ ", la cui area operativa era quella del Mar Rosso, dell'Oceano Indiano e del Golfo Persico. Piri ne fu messo a capo nel 1547. Nel 1548 con una flotta di 60 navi tolse, in una dura battaglia, due castelli allo sceicco di Aden, collaboratore dei Portoghesi, sottraendo a questi ultimi il controllo della regione. La successiva mira dell'ammiraglio fu quella di espellere i Portoghesi dalle acque ottomane e di assicurare alla Sublime Porta il controllo dello Stretto di Ormuz e del Golfo Persico.

All' inizio dell'estate del 1552 con una flotta di trenta navi prese ai Portoghesi il castello di Muscat e sconfisse una flotta di settanta vascelli nemici. Tuttavia, poiché le sue forze avevano subito danni considerevoli, Piri lasciò l'assedio di Ormuz e si diresse su Basra fra continue battaglie e perdite di naviglio. Ma il vecchio ammiraglio non soltanto non ricevette qui aiuto dal governatore ottomano e non fu neppure ammesso nella città; dovette anche affrontare la difficoltà di numerose defezioni tra $\mathrm{i}$ suoi marinai. Nel 1553 si destreggiò per ritornare a Suez, con le tre navi che ancora gli restavano, per non essere intrappolato dai Portoghesi nello stretto di Ormuz. Non si aspettava che a Suez, dove giunse con due navi, avrebbe sofferto la maggiore ingiustizia della sua esistenza, pagandone il prezzo con la vita!

Durante la sua lunga assenza da Istanbul, -come spesso accade in questo mondo a chi non si abbassa al livello dei piccoli intrighi-, non poche trame si erano intessute alle sue spalle. Non gli valse il suo glorioso passato. Non gli giovò quanto egli aveva fatto per la gloria e la grandezza dell' Impero. Persino le sue recenti vittorie furono dimenticate. Accusato ingiustamente di avere abbandonato la flotta e di essere fuggito, Piri Reis fu giustiziato -meglio sarebbe dire martirizzato- con la decapitazione al Cairo nel 1554 per ordine di un firmano giunto da Istanbul, dietro macchinazioni del governatore di Basra ${ }^{13}$. Aveva oltrepassato gli ottant'anni di età.

Oltre alla sua lingua nativa, Piri conosceva il greco, l'italiano, lo spagnolo, il

12 Ufficiale generale della marina dell'India.

13 Ediz. del Kitab-i Babriye cit., pp. 17-18. 
portoghese. Ai suoi tempi l'arte della navigazione marittima si trasmetteva verbalmente attraverso l'esperienza. Soltanto Piri Reis e Seydi Ali Reis (altro uomo di mare del secolo XVI) misero per iscritto le loro conoscenze. Di Piri Reis ci sono pervenute due mappe, rispettivamente del 1513 e del $1528 / 29$, ed un libro, il Kitab-i Babriye (Libro della marina), di cui egli redasse due stesure: la prima nel 1521 , la seconda nel 1525 o 1526.

Egli fa parte di quella fioritura culturale ottomana che contraddistinse gli ultimi lustri del secolo XV e la prima parte del XVI, annovera, tra gli altri, il noto cronista Tursun Beg, raggiunse vere e proprie manifestazioni rinascimentali con Matracki Nasuh, il quale ci ha lasciato nel ms. dell'opera Tarib-i Feth-i Siklos, Estergon, ve Estonibelgrad intorno al 1545, nel museo Topkapi di Istanbul, alcune belle vedute, in miniatura, di città mediterranee, ivi compresa Genova ${ }^{14}$.

La più antica notizia a stampa, in lingua turca, sull'America si contiene in un racconto di Anonimo del tempo del sultano Murad III (1574-1595), pubblicato a Costantinopoli nel 1729/30 sotto il titolo: Ta'rikb-i Hind-i gharbi yakbod badith-i nev (Storia dell'India Occidentale o Nuovo Racconto). E' un insieme di notizie favolose, derivate da testi europei, in particolare da fonti veneziane della seconda metà del Cinquecento ${ }^{15}$.

Nel patrimonio delle tradizioni orali turche non manca un riferimento a Cristoforo Colombo. Lo si legge nella biografia del poeta 'Arif Efendi o 'Arif Mulla (Coxstantinopoli, 1771/72-1849), scritta e publicata a Costantinopoli al principio del secolo scorso da Emin Efendi ${ }^{16}$. Vi si dice che al tempo di Bayazid II (14811512) un cristiano di nome «Kolon» fu a Costantinopoli e chiese l'aiuto del sultano per andare alla scoperta di un «nuovo mondo»; ma gli esperti di corte respinsero il progetto e costrinsero Colombo a ripartire. Sappiamo quasi per certo che Colombo fu a Chio, governata dalla Maona genovese, nel 1474 o 1475; però non ci sono nessuna prova e neppure altra notizia di una sua presenza a Costantinopoli: tanto più che $\mathrm{i}$ termini cronologici non coincidono. Al tempo di Bayazid II Colombo fu prima in Portogallo, poi in Castiglia.

$\mathrm{Ci}$ chiediamo invece se vi sia una correlazione tra questa notizia, trasmessa da una tradizione orale e raccolta da Emin Efendi, ed i referimenti a Colombo, più storicamente concreti, di Piri Reis, di cui diremo. E' cosa possibile: è possibile cioè che già in epoca colombiana il nome del grande Genovese e l'eco della sua impresa circolassero, con deformazioni tipiche dell'oralità, nel mondo della marineria e della cultura ottomane.

La prima mappa di Piri Reis fu disegnata a Gelibolu nel 1513, nel mese di «muharrem» (tra il 9 marzo ed il 17 aprile) e presentata al sultano Selim I, conquistatore dell'Egitto, nel 1517. Essa è però soltanto una parte di un più ampio mappamondo che nella sua originaria interezza comprendeva l'intero orbe terrac-

14 EsIN ATIL, The age of Sultan Suleiman the Magnificent, New York, 1987, pp. 85-87. Cfr. anche G. Pistarino, Variazioni sul tema colombiano, in «Medioevo. Saggi e rassegnew, 12, 1983, pp. 153-168.

15 Cf. L. BONELLI, La scoporta dell'America secondo un'opera turca del secolo decimosesto, 1892; E. RossI, Scritti turcbi su Cristoforo Colombo e la scoperta dell'America, in «Studi Colombianiw, II, 1952, Pp. 563-566.

16 Cfr. M. KeMal, Son asir turk sairlei, Istanbul, 1930, pp. 566; E. ROSSI cit., p. 566. 
queo allora conosciuto e descritto anche, successivamente, dallo stesso Piri nel Kitab-i' Babriye, con i suoi sette mari, mentre il frammento pervenutoci riguarda solo l'area dell'Atlantico in quella parte del Mar dei Caraibi che era stata recentemente scoperta, più la Spagna, il golfo di Biscaglia, l'Africa occidentale.

Questa carta del 1513 ebbe larga diffusione tra gli uomini di mare turchi e venne copiata più volte ad uso della flotta ottomana. Ciò spiega la rinomanza del nome di Colombo nell'ambiente marinaro osmanlo e l'origine della tradizione addirittura sulla sua presenza a Costantinopoli, con la trasposizione al Sultano dell'episodio, riferito da Piri Reis, della richiesta ai governanti genovesi di una spedizione esplorativa verso un "nuovo Mondo".

Questo importante frammento della carta del 1513 fu scoperto nel Topkapi Saray di Istanbul nel 1929 da Halil Ethem Eldem, direttore del museo, mentre, in occasione di lavori di restauro, esaminava i libri e le carte del palazzo, insieme con lo studioso tedesco A. Deismann, autore, successivamente, dello studio Forschungen und Funde im Serai, Berlin-Leipzig, 1933. Un altro noto studioso tedesco, l'orientalista Paul Kahle, allora di passaggio a Costantinopoli, ritenne che la mappa, di cui il frammento doveva fare parte, fosse la copia di un originale, tracciato da Cristoforo Colombo nel 1498, anzi che fosse la mappa stessa presentata da Piri Reis al Sultano d'Egitto nel 1517.

Il Kahle era già autore di un' opera su Piri Reis Babriye. Das türkisches Segelhandbuch für das Mitteländisches Meer von Jabre 1521, Berlin-Leipzig, 1926, dove nel primo volume riportava in parte il testo dell'Autore, nel secondo ne dava la traduzione. Aveva ripreso l'argomento in sede critica con il saggio Piri Reis und seine Babrije in "Beiträge zur historischen Geographie, Kulturgeographie, Ethnographie und Kartographie vornehmlich des Orients», Leipzig-Wien, 1929, pp. 60-76. Il problema della carta di Piri Reis venne da lui affrontato pubblicamente nel XVIII Congresso degli Orientalisti, tenutosi a Leida nel 1931: la sua comunicazione fu tradotta in italiano da G. Levi Della Vida (Impronte colombiane in una carta turca del 1513, in «La Cultura Moderna», X.1, Milano-Roma, 1931, pp. 775-785), come pure in spagnolo (Un mapa de América becho por el turco Piri Re'is en el año 1513, basándose en un mapa de Colón y en mapas portugueses, in "Investigación y Progreso", V, Madrid, núm. 12, diciembre 1931).

L'argomento fu trattato dal Kahle in un saggio del 1932 (Die verschollen Columbus-Karte von America von Jabre 1498 in einer türkischen Weltkarte von 1513, in "Vorlesungen und Fortschritte», Berlin-Leipzig, 1932) e ripreso nel 1933 ( $A$ lost map of Columbus, in "Geographical Review», XXIII, pp. 621-638) e nel 1935 (The lost Columbus map of 1498, in "Aligarh Muslim University Journal», II).

Ma esso, che può già trovare un indiretto preludio, come filone di studi, in un opuscolo di A. D'Avezac del 1886 (Note sur une mappamonde turke de XVle siècle conservée à la Bibliothèque de Saint-Marc à Venise, Parigi, Société de Géographie) ${ }^{17}$,

17 Tra le numerose pubblicazioni uscite nel 1892, in occasione delle celebrazioni del quarto centenario della scoperta dell'America, non è mancata la presenza della lingua turca. Uscì a Costantinopoli un volume: Ta'rikbi-i keshf-i Ameriqa, che non è però un'opera originale, ma una traduzione. Cfr. Otbmanli Mü'ellifleri, Istanbul, 1924; IBRAHIM HAKKI, Topkapi Sarayinda deri izzerine yapilmis eski haritalar, Istanbul, 1936; E. RossI cit., p. 563. 
suscitò, di fronte al Kahle, una serie d'interventi, sia puramente informativi, sia in sede di discussione critica. Si vedano specificamente E. Oberhummer (Eine Türkische Karte zur Enteckung Amerikas, in "Anzeiger der Akademie der Wissenschaften in Wien", Phil. Hist. Klasse, 68, 1931, pp. 99-112); gli scrittori turchi d'informazione, come Hüseyin Yurdaydin (Map drawn by Piri Reis, one of the Turkish Geograpbers, in "Illustrated London News», 25 febbraio 1932) e Yusuf Arçura (Map drawn by Piri Reis, in "Illustrated London News», 23 luglio 1932); Paolo Emilio Taviani (A proposito del rinvenimento a Istanbul di una carta colombiana, "Il Nuovo Cittadino", Genova, gennaio 1932); Roberto Almagià (Il mappamondo di Piri Reis e la carta di Cristoforo Colombo del 1498, in "Genova», Genova, marzo 1934, ed in "Bollettino della Società Geografica Italiana», Roma, 1934, pp. 442-449); E. Oberhummer (An early turkish map of America and the last map of Columbus, in "Actas y trabajos científicos. XXV Congreso Int. de Americanistas, La Plata, 1932», Buenos Aires, 1934, II, pp. 283-286; Id., Eine Karte des Columbus in türkischer Überlieferung, in «Mitteilungen der Geographisch Gesellschaft in Wien», LXXVII, 1934, núm. 7-9, pp. 115-117); K. Kretschmer (Die verschollene Kolumbuskarta von 1498 in einer türkischen Weltkarte von 1513, in "Petermann's Mitteilungen», Gotha, 80, 1934, pp. 48- 50); P. Revelli (Cristoforo Colombo e la scuola cartografica genovese, Roma, 1937); P. Braunlich (Zwei türkischen Weltkarten aus dem Zeitalter der grossen Entdeckungen, in «Berichte über die Verhandlungen der Sächs. Ak. der Wiss. zu Leipzig», Phil.-Hist. Klasse, 89, I, 1939; Id., Die türkischen Weltkarten von 1513 und 1528, in "Forschungen und Fortschritte», XIV).

Naturalmente non sono mancati, dopo la scoperta di questa mappa di Piri Reis al Topkapi Sarayi, i lavori in lingua turca, nel corso degli anni trenta. Segnaliamo specificamente Yusuf Arçura (Piri Reis haritasi bakkinda izabname-La carta di Piri Reis, T.T.K. Yayinlari, 1, Istanbul, 1935); Alpagut Aydar-F. Kurtoğlu, Piri Reis, Kitabi Babriye, T.T.K. Yayinlari, 2, Istanbul, 1936); Miss Afet (Bir Türk Amirali XVI. asrin biiyük ceografi: Piri Reis - Un ammiraglio geografo turco del XVI secolo: Piri Reis, in «Belleten», I.2, Ankara, 1937); Hamid Selen Sadi (Piri Reis'in Simali Amerika Haritasi - La carta del Nord America di Piri Reis, in "Belleten", I.2, Ankara, 1937). Opera notevole, nonostante il valore disuguale, è il volume di Ibrahim Hakki (Topkapi Sarayinda deri üzerine yapilmis eski baritalar - Antiche pergamene del Palazzo di Topkapi, Istanbul, 1936), che contiene notizie varie, polemiche, excursus storici e geografici.

La carta di Piri Reis è tornata successivamente all'attenzione degli studiosi: di nuovo con R. Almagià (Il mappamondo di Piri Reis e la carta di Colombo del 1498, in "Actas del XXVI Congreso Intern. de Americanistas, Madrid, 1948», Madrid, 1950, vol. II, pp. 32-41); con Ettore Rossi (Scritti turchi su Cristoforo Colombo e la scoperta dell'America, in "Studi Colombiani», II, Genova, 1952, pp. 563-566); con A. Afetinan (Piri Reis'in Amerika Haritasi (1513) - La più antica mappa dell'America (1513), Ankara, 1954); con E. Reguerra Sierra (El mapa de Piri Reis. Examen y valoración del pintoresco, raro y desconcertante portulano ejecutado por el almirante turco Piri Reis en 1513, y presumiblemente la primera carta geográfica turca que se refiere al bemisferio de Colón, in "Revista Geográfica Americana», Buenos Aires, XXXVIII, 1954, pp. 43-46); di nuovo con P. Kahle (Piri Re'is der türkische Seeman und 
Kartograph, in "Wiessenschaften Annals", Wissenbourg, V, 1956, pp. 862-872); con I.O. Bignardelli (Il navigatore Piri Re'is invece di Colombo avrebbe scoperto il Nuovo Mondo, in "Genova Municipale», Genova, 1961, pp. 14-23); con A. Afetinan (The oldest map of America, drawn by Piri Reis. Life and works of a Turkish Admiral, traduz, dal turco di Leman Yolaç, Ankara, 1964; Id., Life and works of Piri Reis, traduz. dal turco di Leman Yolaç e Engim Uzmen, Ankara, 1975); di nuovo con P.E. Taviani ( Si dice che un genovese infedele di nome Colombo abbia scoperto il luogo", in "Liguria», XL, giugno- luglio 1973, n. 6-7, pp. 7-11; Id., Cristoforo Colombo. La genesi della grande scoperta, Novara, 1974, ediz. economica Novara, 1982, pp. 158, 179, 220, 222, 447); con K.A. Tonge (The World Map of Piri Re'is, in «Information Bulletin Western Association of Map Libraries", Santa Cruz, june 1976, pp. 4-8); con W. Leitner (Die "Amerikakarte» des Piri Réis von 1513: ein Beitrag zur tïrkischen Kulturgeschichte und zur Kolumbusforschung, in "Blätter für Heimatkunde», Graz, 1981 , n. 55, pp. 69-80).

Vi hanno anche accennato, seppure incidentalmente, Elio Migliorini (Gli studi colombiani nell'opera dei geografi italiani dell'ultimo secolo, in "Atti de II Covegno internazionale di studi colombiani, Genova, 6 e 7 ottobre 1975», Genova, 1977, pp. 17-72, in particolare p. 41), il quale si dichiara d'accordo col Baldacci nel ritenere che l'attività di Piri Reis, in relazione alla sua carta nautica, andrebbe ulteriormente approfondita; ed Alessandro Bausani (L'Italia nel Kitab-i Babriyye di Piri Reis, in "Il Veltro. Rivista della civiltà italiana», XXIII, num. 2-4, marzoagosto, 1979, Le relazioni tra l'Italia e la Turchia, pp. 173-196).

Lo stesso Mustafa Kemal Atatürk, fondatore della Repubblica turca, mostrò grande interesse per la carta di Piri Reis del 1513: la studiò personalmente e nel 1935 ne promosse la riproduzione in facsimile a colori per opera della tipografia di Stato. L'edizione del 1935, dal titolo Piri Reis haritasi hakkinda izabname (Spiegazione sulla carta di Piri Reis), pubblicata ad Istanbul a cura di Yusuf Arçura, presenta la descrizione del testo in turco moderno, tedesco, inglese e francese, e la riproduzione a colori del ms. Una seconda edizione, con le didascalie trascritte in alfabeto turco moderno e tradotte in francese, è stata pubblicata in Piri Reis haritasi (La carta di Piri Reis), Istanbul-Cubuklu, 1966, a cura dell'Ufficio della Marina turca di Navigazione, Idrografia ed Oceanografia. Una terza edizione è uscita nel 1981. Nello stesso tempo, la Società Storica Turca ha redatto il commentario della carta medesima.

Nella biblioteca del Topkapi Sarayi si conserva un'altra carta con la leggenda seguente: «Piri Reis, figlio di Haci Mehmet, fratello del defunto Kemâl Reis. Città di Gelibolu, nell'anno 935». Appare evidente che si tratta di una seconda carta di Piri Reis, prodotta nel 1528-29. Risulta anche che essa è la sesta parte di un originario mappamondo. Presenta l'area settentrionale dell'Oceano Atlantico, le coste del Nord America, dalla Groenlandia alla penisola della Florida. La precisione di questa carta è notevolmente superiore a quella del 1513; ciò dimostra che la scienza nautica era allora in rapido sviluppo. E c'è un interessante riferimento, seppure senza richiamo onomastico, all'impresa de Vasco Nuñez de Balboa, che nel 1513 attraversò l'America centrale e raggiunse il Pacifico: il che comprova il costante vivo interesse di Piri Reis per il progresso delle esplorazioni geografiche. 
Uno dei motivi dell'importanza della carta del 1513 di Piri Reis consiste appunto -sottolinea P.E. Taviani- nel fatto che essa è, come dice lo stesso Piri, il frutto di studi comparativi e deduttivi su molte carte: "Venti carte -cito Taviani-e mappamondi del tempo antico, delle quali alcune dell'epoca di Alessandro il Grande; otto carte delle scuole islamiche, fra le quali una araba delle Indie; una carta portoghese del XV secolo, con le Indie e la Cina disegnate geometricamente, nonché carte indiane e cinesi (...). Riducendo a una sola le scale di tutte queste carte, Piri Reis arrivò alla somma finale». Tale da ritenere la sua mappa corretta ed attendibile "per i sette mari». "Nessuno del secolo attuale -afferma Piri Reis- può dichiarare di avere una carta simile. L'affermazione non è una vanteria. E' tanto corrispondente al vero, in quanto, come l'autore esplicitamente spiega, la sua opera si basa su una carta delle regioni occidentali, elaborata da Cristoforo Colombo" ${ }^{18}$.

Tra le note, che la carta presenta, è particolarmente importante quella che porta il numero 5 e che qui riferiamo integralmente nella versione italiana, presentata da un noto turcologo ${ }^{19}$. Il tenore del testo, per ciò che riguarda il Nuovo Mondo, offre un indubbio aspetto di genuinità, sì che appare credibile la sua attribuzione a qualcuno che l'abbia riferita a Piri Reis per esperienza diretta del mondo d'oltre l'Atlantico, anche se Elio Migliorini, in accordo con l'Almagià, considera assai improbabile che uno schiavo, catturato su nave spagnola nel 1501, « abbia potuto recare con sé una carta, tanto più che la figurazione è molto diversa da quella che ci si aspetterebbe dai viaggi colombiani (...). Probabilmente lo schiavo ha potuto fornire al cartografo qualche elemento, per cui è più opportuno parlare di impronte colombiane», come fa appunto l'Almagià.

"Queste coste si chiamano litorale di Antilya. Sono state scoperte nell'anno 890 dell'era araba ${ }^{20}$. E si racconta che un infedele di Genova, chiamato Colombo, ha scoperto queste contrade. Cadde, cioè, fra le mani di Colombo un libro in cui apprese che ai confini del mare d'occidente, cioè ad ovest, esistevano delle coste e delle isole, ogni genere di miniere e anche pietre preziose. Avendo letto da un capo all'altro quell'opera, raccontò l'uno dopo l'altro questi fatti ai Grandi di Genova e disse loro: "Datemi due navi che vada a trovare quei luoghi...». "Sciocco-gli risposero- il mare d'occidente avrebbe dunque un limite o una fine? $\grave{E}$ avviluppato dai vapori delle tenebre!». Il detto Colombo, visto che non c'era nulla da attendersi dai Genovesi, se ne andò a raccontare la cosa al Re di Spagna. Anche lui gli diede la stessa risposta che gli avevano dato i Genovesi. Ma Colombo insistette tanto che il Re di Spagna gli diede due navi, curò che fossero bene armate ed equipaggiate e

18 P.E. Taviani, "Si dice cbe un genovese infedele, dal nome Colombo, abbia scoperto il luogos, in «Ligurian, XL, num. 6-7, giugno-luglio 1973, Pp. 7-12.

19 Alessandro Bausani, L'Italia nel «Kitab-i Babriyye» di Piri Reis, in «Il Veltro, Rivista di civiltà italianaw, XXIII, n. 2- 4, marzo-agosto 1979, pp. 174-1 76. Esiste la precedente versione italiana di P.E. TAVIANI, «Si dice che un genovese infedele dal nome Colombo abbia scoperto il luogon, in aLigurian, XL, num. 6- 7, giugno-luglio 1973, Pp. 10-12.

20 L'anno 890 dell'Egira corrisponde al periodo dal $1 .^{\circ}$ gennaio 1485 al 7 gennaio 1486 . Nitra lettura del testo originale dà invece l'anno arabo 870, corrispondente al periodo dal 24 agosto 1465 al 13 agosto 1466: cfr. $\Lambda$. AFETINAN, Life and Works cit., p. 21. 
disse: "O Colombo, se è come tu dici, io ti faccio capitano di quei luoghi», e lo mandò nel mare d'occidente. Il defunto Gazi Kamâl possedeva uno schiavo spagnolo, il quale schiavo raccontava a Kamâl Reis che era andato per tre volte in quel paese con Colombo, e diceva: "Arrivammo dapprima allo stretto di Ceuta (Gibilterra), poi, dopo aver percorso quattromila miglia in direzione fra ovest e sudovest, ci trovammo di fronte un'isola, ma le onde del mare spumeggiavano sempre meno (cioè il mare si calmò); la stella del nord, che i marinai consultando la bussola chiamano «la Stella», scomparve a poco a poco». Aggiungiamo che le stelle in quel paese non si mostrano nella posizione che ci è familiare, $\mathrm{ma}$ in un'altra. Intanto gettarono l'ancora davanti a un' isola che vedevano di fronte a loro. Ma sopraggiunsero gli abitanti dell'isola e lanciarono frecce, impedendo loro di sbarcare e di domandare informazioni. Uomini e donne lanciavano frecce. Le punte di queste frecce erano fatte di spine di pesce. Gli abitanti erano tutti nudi e molto... (testo indecifrabile). In breve videro l'impossibilità di sbarcare in questa isola e andarono dall'altra parte della medesima, dove videro una barca che, alla loro apparizione, virò e sbarcò a terra i suoi occupanti. Gli Spagnoli corsero a impadronirsi della barca e vi constatarono la presenza di carne umana. Si trattava dunque di persone che, andando d'isola in isola, facevano la caccia agli uomini per nutrirsi delle loro carni.

Il detto Colombo vide un'altra isola e vi si recò. Vi constatò la presenza di grossi serpenti. Temendo di sbarcarvi, andò a un'altra isola dove, gettate le ancore, rimase sette giorni. Gli abitanti di quell' isola, visto che quel vascello non causava loro alcun danno, presero dei pesci che portarono a bordo delle loro imbarcazioni. Gli occupanti del vascello, soddisfatti, diedero loro in cambio perline di vetro (sïrşa boncuk). Avevano infatti appreso dai libri che le perline di vetro erano molto apprezzate in quei paesi. La vista di quelle perline fece sì che [gli indigeni] portassero un maggior numero di pesci, e gli altri dettero loro ancora più perline. Questi (gli Spagnoli) un giorno videro ornamenti d'oro sul braccio di una donna. Presero quei gioielli in cambio di perline di vetro e dissero: "Andate a cercarci dell'oro, noi vi daremo delle perline!» $\mathrm{Al}$ che gli altri portarono una più grande quantità d'oro. Infatti le loro montagne nascondevano delle miniere d'oro. Un altro giorno videro delle perle in mano a un indigeno e scambiarono anche quelle con perline di vetro, vedendo la qual cosa gli indigeni portarono perle in gran quantità. Le perle si trovavano sul litorale di quell' isola a due braccia di profondità. Essi (gli Spagnoli) imbarcarono una gran quantità di legno di baqqam (che dà una tintura rossa) e portarono con sé due abitanti del luogo e li presentarono al re di Spagna. Ma il detto Colombo ignorava la lingua di quegli uomini: così le loro transazioni erano fatte a mezzo di segni. Dopo questo viaggio il re di Spagna inviò dell'orzo agli indigeni e dei preti (papaz) che insegnarono a seminare e a raccogliere e li fecero entrare nella loro religione, perché in certo modo non ne avevano nessuna. Gli indigeni andavano in giro nudi come animali e si coricavano per terra. Ora questi paesi sono aperti a tutti e sono conosciuti. I nomi che designano le dette isole e i litorali Colombo li diede perché fossero conosciuti sotto quei nomi. Colombo era anche un grande astronomo (müneccim). I litorali e le coste che figurano su questa carta sono presi dalla carta di Colombo...». 
«Nessuno nel secolo presente possiede una carta simile a questa, elaborata e disegnata dall'umile sottoscritto (bu faqir). La presente carta è il prodotto degli studi comparativi e deduttivi fatti su venti carte e mappamondi - cioè esiste una carta fatta all'epoca di Alessandro Magno, comprendente tutta l'ecumene, tipo di carta che gli Arabi chiamano ca'feriyye- e anche su otto simili ca'feriyye ${ }^{21}$, su una carta araba delle Indie, come anche su una carta disegnata recentemente da quattro Portoghesi e dove i paesi di Sind, Hind e Cina sono disegnati con mezzi geometrici, e anche su una carta di Colombo, elaborata per l'emisfero occidentale...»

Pure con tutte le confusioni e le incertezze che possono nascere dagli scambi e dalle sovrapposizioni tra diversi viaggi e da ricordi lontani, come anche dalla trasmissione orale (dallo schiavo spagnolo a Kemâl Reis e da quest'ultimo a Piri Reis), le notizie riferite dallo schiavo trovano sostanziale riscontro nei memoriali dei viaggi colombiani, a partire dal "Giornale di bordo" del primo viaggio. L'osservazione sulla stella polare si può accostare a quanto l'Ammiraglio scrive il 17 settembre 1492 ( «sembra che sia la stella a cambiare la sua posizione, non gli aghi») ed il 30 successivo ( «è chiaro che questa stella si muove come le altre stelle e gli aghi sono sempre sinceri»), il 21 novembre ( «la stella del Nord era alta come in Castiglia»), e, nel ritorno, il 3 febbraio 1493 ( «la stella del Nord sembrava alta come al capo San Vincenzo») ${ }^{22}$. Soprattutto ci vengono a mente le osservazioni dell'Ammiraglio nella relazione del terzo viaggio, quando egli scrive che, superando le cento leghe a ponente delle Azzorre, scoprì che «la estrella del Norte escrive un çírculo, el cual tiene en el diámetro cinco grados, y estando las Guardas en el braço derecho, estonçes está la estrella en el más baxo y se va alçando fasta que llega al braço izquierdo; y estonçes está cinco grados, y de allí se va abaxando fasta llegar a bolver otra vez al braço derecho (...). Fallé allí que, en anocheçiendo, tenía yo la estrella del Norte alta cinco grados, y estonces las Guardas estavan ençima de la cabeça; y después, a la media noche, fallava la estrella alta de diez grados, y en amaneciendo, que las Guardas estavan en los pies, quince» ${ }^{23}$.

Ci richiamano al "Giornale di bordo" del primo viaggio colombiano le notizie sugli indiani, che vanno tutti nudi ed ora fuggono alla vista degli spagnoli, ora li accolgono amichevolmente, con il baratto tra gli oggetti d'oro dei nativi e le cianfrusaglie dei nuovi venuti; gli accenni alle punte delle lance fatte di spine di pesce; il prelievo di indigeni già nella prima esplorazione (ma più di due) per condurli alla corte di Spagna; le denominazioni date dall'Ammiraglio alle nuove

21 Sui vari tipi di carte arabe cfr. K. Miller, Mappae Arabicae. Arabiscbe Weltund Länderkarten des 9-13 Jbdts, in arabischer Urschrift, Lateinischer Transcription Und Übertragung in neuzeitlicbe Kertenskizzen, Stuttgart, 1926-1931. 162.

22 Il giomale di bordo di Cristoforo Colombo, traduz. di AlDo BraibantI, Milano, 1960, pp. 11, 18, 70,

23 Consuelo Varela. Cristóbal Colón. Textos y documentos completos. Relaciones de viajes, cartas y memoriales, II ediz. Madrid, 1984, p. 212. «la stella del Nord descrive un cerchio, che ha nel diametro cinque gradi, e quando le Guardie sono al braccio destro, allora la stella si trova nel punto più basso, quindi va alzandosi sino a che giunge al braccio sinistro; allora è a cinque gradi, e di lì si va abbassando sino a tornare nuovamente al braccio destro (...). Scoprii lì, quando si fece buio, che la stella del Nord era alta cinque gradi, e allora le Grardie erano sopra la mia testa; dopo, a mezzanotte, la stella era a dieci gradi, e sul far del giorno le Grandie erano ai piedi, ai quindici». 
terre. Ed anche la presenza di serpenti in un' isola trova riscontro in quanto Colombo scrive il 16 ottobre 1492 sul «ragazzo» che gli disse «di avere visto un grosso serpente" nella Ferdinandina (Long Island), e sui serpenti che egli ed i suoi uomini il 21 ottobre, Martín Alonso Pinzón il giorno dopo, uccisero nell'Isabela (Crooked Island). E l'Ammiraglio aggiunge: "Credo che in queste lagune ci siano molti serpenti del genere»"

L'isola, in cui l'Ammiraglio ed i suoi uomini rimasero sette giorni, potrebbe ravvisarsi ancora nella Hispaniola durante il primo viaggio, nella quale Colombo sostò dal pomeriggio del 19 ottobre 1492 alla mezzanotte del 24, mentre poi trascorse tutto il 25 in navigazione; oppure in Santa Maria di Guadalupe, dove, nel corso della seconda spedizione, egli ed i suoi si fermarono per circa sei giorni, secondo la testimonianza di Michele da Cuneo: più precisamente dal 3 al 10 novembre $1493^{23}$.

Se invece di «sette giorni» si legge «diciassette giorni», come nell'edizione del testo riportata da P.E. Taviani ${ }^{26}$, ci si può riferire al periodo tra il 28 ottobre ed il 12 novembre 1492, trascorso da Colombo in Cuba ${ }^{27}$, o addirittura, se lo spagnolo era imbarcato sulla «Pinta» di Martín Alonso Pinzón, alla cosiddetta defezione di quest'ultimo dal 21 dicembre 1492 al 6 gennaio 1493, cioè sedici giorni, mentre il Pinzón fece giurare ai suoi uomini che si erano fermati solo sei giorni ${ }^{28}$.

Ugualmente al secondo viaggio bisogna rifarsi per le prove concrete dell'esistenza dei cannibali, sebbene l'Ammiraglio vi accenni ripetutamente, per sentito dire, nel «Giornale di bordo» della prima navigazione; ma non volle mai prestarvi fede, neppure quando egli ed i suoi, il 29 novembre 1492, videro dei teschi dentro a canestri ( «l'Ammiraglio suppose che dovessero essere le teste dei capostipiti») o quando alcuni indigeni gli mostrarono, il 17 dicembre, le cicatrici dei bocconi dai loro corpi strappati a morsi dai Cannibati o Cannibali; «ma l'Ammiraglio non lo credette». Forse cominciò a ricredersi nel colloquio con il re di Bohio (Española), Guacanagarí, il 26 dicembre, dopo il naufragio della «Santa Maria», e poi quando, nel viaggio di ritorno, il 13 gennaio 1493, ricevette a bordo un indigeno "che all'aspetto era il più brutto di quanti avesse visto" e "pensò che dovesse essere

\footnotetext{
24 Il giornale di bondo, cit., p. 43.

25 Cristoforo Colombo, l viaggi dopo la uscopertan, introduzione di Gino BARBIERI, Cassa di Risparmio di Verona, Vicenza e Belluno, Verona, 1985, pp. 163-181.

26 P.E. TAviani, aSi dice cbe un genovese infedeles cit., p. 12.

27 Si tenga tuttavia presente che già nel primo viaggio Colombo mostra ad un certo momento di essere convinto che Cuba fosse il continente tanto ricercato, e che Piri Reis lo seguì in questa tesi. In realtà, tuttavia, l'Ammiraglio rivelà qualche incertezza. Il 24, 26 e 28 ottobre, il 14 novembre, il 12 dicembre 1492 ed il 6 gennaio 1493 egli parla di Cuba come di un isola, mentre il 2 novembre 1492 la considera un continente, ed il 5 dicembre leggiamo: «Siccome il vento tirava da nord-est e da nord, decise di lasciare Cuba o Juana, che fino ad allora aveva considerato come terra ferma..... Nell'uno e nell'altro caso noi disponiamo però soltanto del testo del «Giornale di bordow, tramandatoci dal Las Casas, e che non sappiamo fino a che punto sia fedele all'originale. Oppure la convinzione si radico in Colombo gradualmente, dopo che egli raggiunse quella terra? Od ancora, soltanto lentamente Colombo si rese conto che, in base alle capitolazioni di Santa Fe, i privilegi, concessigli dai suoi sovrani, erano vincolati al fatto che egli raggiungesse il continente al di là dell'Oceano e non semplicemente delle isole? Nella lettera del 15 febbraio 1493, da bordo della «Niña», egli definisce Cuba come isola.

28 Il giornale di bondo cit., pp. 145-148.
} 
di quei Caribi che mangiano gli uomini», oppure quando, il 15 gennaio, riferisce che si diceva che gli abitanti delle isole Carib e Matinino [Martinica] mangiassero carne umana.

In realtà, tuttavia, ancora al secondo viaggio ci si deve rifare per le prove concrete dell'esistenza dei cannibali: quando nella Guadalupe, nel novembre 1493, si trovarono nelle capanne degli indigeni «quattro o cinque ossa di braccia e gambe umane", secondo la testimonianza di Diego Álvarez Chanca, e si ebbe notizia dei barbari costumi dei Caribi, e quando, secondo la testimonianza di Michele da Cuneo, gli spagnoli diedero la caccia ad una canoa in cui erano prigionieri due indiani evirati ${ }^{29}$.

E' nota l'ansiosa, immediata ricerca dell'oro, già nei primi sbarchi nelle terre d'oltremare, mentre le prime perle delle Indie occidentali vennero trovate dagli spagnoli a metà agosto 1498 , sulle coste del Golfo di Paría ${ }^{30}$, quando alcune canoe si avvicinarono alle caravelle colombiane: gli uomini portavano perle attaccate ai polsi; a loro volta, le donne del paese avevano collane fatte di grani, alternati a perle bellissime ${ }^{31}$. Sempre nel terzo viaggio Colombo trovò, nei paraggi del golfo di Paría, le belle foreste di quel legno di brasile che forniva ai tintori di lusso l'ineguagliabile colore del rosso cupo, sebbene il nome di "puerto Brasil», assegnato dall'Ammiraglio all'attuale Jacnel Bay, nel settembre del 1494, farebbe supporre la scoperta dell'esistenza della preziosissima spezia anche nel nuovo mondo già nel corso del secondo viaggio ${ }^{32}$.

Una notizia interessante, che non sappiamo donde sia stata ricavata da Piri Reis, ma che è passata nella storiografia successiva, riguarda il primo tentativo effettuato da Cristoforo Colombo presso il governo genovese, mentre il grande cartografo turco non accenna ad un soggiorno lusitano dell'Ammiraglio, seppure Piri accenni spesso ai Portoghesi.

Circa le due navi, che furono assegnate dal re di Spagna a Colombo per la sua impresa, Piri Reis non si discosta dal vero, giacché furono effettivamente due le caravelle fornite alla spedizione, per imposizione reale, dalla comunità di Palos, mentre la capitana della flotta, la nao "Santa Maria», venne concessa in affitto da Juan de la Cosa. E così pure furono soltanto due le navi che ritornarono in patria, dopo il naufragio della "Santa Maria» alla Hispaniola nel Natale del 1492. Risponde ugualmente al vero la concessione del titolo di Ammiraglio [del Mare Oceano] a Cristoforo Colombo, sancita dalle capitolazioni di Santa Fe. Piri Reis è bene informato: così sa del trattato di Tordesillas del 1494; è al corrente delle imprese che portarono i Portoghesi alla scoperta del Capo di Buona Speranza nel 1487-88, ed all' approdo a Calicut nel 1497; conosce le mappe portoghesi e le scoperte anteriori al 1508 sulle coste sud-americane per opera di Amerigo Vespucci, Pinzón, Juan de la Cosa.

\footnotetext{
29 Cristoforo Colombo, I viaggi dopo la uScopertan cit., pp. 122, 165.

30 Accenni alle perle si leggono nel «Giornale di bordo» del primo viaggio, al 26 e 28 ottobre, al 4, 12 e 16 novembre 1492, ma Colombo ed i suoi non ne vennero in possesso né ritrovarono ostriche perlifere.

31 JaCQubs Heers, Cristoforo Colombo, traduz. di Elina Klersy ImberCiADOR, Milano, 1983, p. 542.

32 J. HeErs cit., p. 491.
} 
Viceversa, presso Piri Reis il Nuovo Mondo non viene definito con l'appellativo di Cipango o Catai, alla maniera di Colombo, o con quello successivo, e più comune, di Indie, Indie Nuove, Indie Occidentali, od anche Terra degli Antipodi, ma con quello di Antilia o Antilla, l'isola leggendaria vagheggiata dagli uomini del medioevo, che però Piri Reis considera terraferma: è qui rilevante l'analogia con la tesi professata da Cristoforo Colombo a proposito di Cuba nel corso del secondo viaggio, quando egli, il 21 giugno 1494, sulla caravella «Niña», fece giurare ai suoi uomini «che quella era terraferma, per la conformazione e le notizie che ne aveva e per $\mathrm{i}$ nomi delle popolazioni delle varie province e in special modo della provincia di Mangow. Il mondo turco, a diretto contatto con le Indie orientali e con l'Estremo Oriente, più di quanto potessero esserlo i paesi cristiani dell'Occidente, non si lasciò sedurre, già per tempo, dalla convinzione colombiana di avere raggiunto il Catai di Marco Polo. Preferì credere che l'Ammiraglio «infedele" avesse finalmente toccato una meta favolosa, invano sperata nel corso di un millennio ${ }^{33}$.

Altre note della carta del 1513 ricordano i genovesi o Cristoforo Colombo. La XIII, apposta nei pressi delle Azzorre, dice: "Una kïke genovese, venendo dalle Fiandre, fu coinvolta in una tempesta. Spinta dall'uragano, arrivò a queste isole, e così esse furono note». La XXI, che si riferisce evidentementea ad Antonio da Noli: "L'ammiraglio di questa caravella si chiamava Messir Antonio il Genovese, ma egli emerse in Portogallo. Un giorno la suddetta caravella incontrò un temporale e fu spinta su quest' isola [di Capo Verde]. Vi trovò molto zenzero». La XXII: "Questo mare è chiamato il Mare Occidentale, ma i navigatori franchi lo chiamano il Mare di Spagna. Fino ad ora era noto con questi nomi, ma Colombo, che lo aprì, e fece conoscere queste isole, ed i Portoghesi, infedeli, che hanno aperto la regione di Hind, hanno insieme convenuto di dare un nuovo nome a questo mare. Lo hanno chiamato Ovo Sano [Oceano], vale a dire «uovo intero". Anteriormente si riteneva che il mare non avesse fine né limite; che all'altra estremità fosse la tenebra. Ora che si è visto che questi mari sono circondati da una costa, perché è simile ad un lago, l'hanno chiamato Ovo Sano".

Piri c'informa, in una leggenda della carta, -come s'è visto- che egli disegnò questa mappa desumendola da altre carte e mappe mundi, da carte arabe, da recenti carte portoghesi sulle contrade di Hindi, Sindi e Cina, da una mappa delle regioni occidentali, tracciata da Cristoforo Colombo, riducendo il tutto geometricamente in una unica scala. Il fatto che egli sia riuscito a produrre una tale opera con le informazioni contenute nelle carte prese a modello (isole e coste) e redatte in lingue diverse (arabo, spagnolo, portoghese, greco, castigliano) e con l'aggiunta delle esperienze personali dell' autore, dimostra -sottolinea P.E. Taviani- la grandissima abilità di Piri Reis. E' una carta superiore alle precedenti a noi note non solo per la maestria del tracciato, ma anche per la sua praticità ai fini dell'arte nautica ${ }^{34}$.

33 G. Pistarino, Variazioni sul tema colombiano, in *Medioevo. Saggi e rassegnew, 12, 1988, pp. 153-168. Sulla notizia della proposta colombiana del progetto dell' impresa atlantica alla Republica di Genova cfr. P.E. TAviANI. Il diritzo-dovere dello storico di fermarsi su alcuni temi colombiani a wn'altermativa di differenti iposesi, in «Atti del IV Convengo internazionale di studi colombiani, Genova, 21-23 ottobre 1985n, Genova, 1987, pp. 46-49.

34 P.E. Taviani, Cristoforo Colombo cit. Pp. 158, 179, 220, 222, 447. 
Come ancora rileva Taviani abbiamo qui, nella carta del 1513, una nuova prova, del tutto oggettiva, della genovesità dell' Ammiraglio del Mare Oceano. E' invece inesatto l'anno della scoperta. Ma se si rettifica la lettura in 896 , cioè nel periodo dal 14 novembre 1490 al 3 novembre 1491 , come sembra suggerire lo stesso Taviani, l'errore di Piri Reis si riduce a pochi tempi, dovendosi oltre tutto tenere presente che in realtà la scoperta dell'America avvenne non il $12 \mathrm{ma}$ il 22 ottobre, secondo l'odierno calendario gregoriano (il 12 ottobre l'approdo sarebbe stato assai difficile a causa dell'anticiclone delle Azzorre, come sostiene José Luis Comellas) ${ }^{35}$.

Per quanto riguarda la carta di Colombo, che dovette essere utilizzata da Piri Reis, si trattò probabilmente di una delle copie fatte in Ispagna sulla base di un originale allegato dall'Ammiraglio in una lettera ai suoi sovrani dall' Hispaniola il 31 agosto 1498 e che, divulgato, contro la sua volontà, in più esemplari, facilitò $i$ cosiddetti "viaggi andalusi» del 1499-1500. Scrive Colombo: «Entre tanto que vengan a notiçia d'esto d'estas tierras que hagora nuebamente e descubierto, en que tengo assentado en el ánima que allí es el Paraíso Terrenal, irá el Adelantado con tres navíos bien ataviados para ello, a ver más adelante, y descubrirán todo lo que pudieren hazia aquellas partes. Entre tanto yo enbiaré a Vuestras Altezas esta escriptura y la pintura de la tierra, y acordarán lo que en ello se deva fazer y me enbiarán a mandar». Consuelo Varela ritiene che questa sia la carta di cui prese visione Alonso de Hojeda e che fu un incentivo alla sua spedizione insieme con Amerigo Vespucci, nel 1499, fino a Paría, dando base alla famosa carta del Vespucci medesimo ${ }^{36}$. Taviani giustamente suppone che "la carta delle regioni occidentali, a cui Piri Reis si è ispirato, fosse una delle carte in uso sulle navi spagnole»" ${ }^{37}$, anche se evidentemente suggerite dalla carta colombiana del 1498.

Ancora: quando Piri Reis riferisce che il defunto Gazi Kemâl aveva uno schiavo spagnolo, il quale narrò a Kemâl Reis di essere stato tre volte in quelle terre oltre l'Oceano insieme a Colombo, a chi personalmente allude? Come ricorda P.E. Taviani, «nel 1501 i marinai turchi sostennero una violenta battaglia navale nel Mediterraneo occidentale. Catturarono alcuni bastimenti spagnoli, in uno dei quali trovarono vari oggetti e prodotti provenienti dall'America. Nell'opera Babriye così scrive Piri Reis: «Sulle navi nemiche, catturate da noi nel Mediterraneo, abbiamo trovato un berretto fatto di piume di questi pappagalli ed una pietra simile al diasprom. Probabilmente in quella medesima circostanza fu preso lo spagnolo che raccontò a Kemâl Reis di essere stato tre volte in quella lontana terra: quindi a cominciare dal primo viaggio colombiano. Era difficile che mentisse, perché ancora Colombo era in vita: anzi stava preparando la sua quarta esplorazione.

In realtà, non dovette mancare, e non mancò, qualche uomo di mare che partecipò ai primi tre viaggi dell'Ammiraglio, od anche a tutti quattro. Citiamo, a titolo di esempio, lo spagnolo Bartolomé Garcia, del cui intervento alle prime tre 42.

35 Laura Balletto, Il cielo di Colombo, in «Columbus 92w, v, num. 11-12, novembre-dicembre 1989, pp.

36 C. Varela, Cristóbal Colón. Textos y documentos completos. Relaciomes de viaje, cartas y memoriales, II ediz., Madrid, 1984, p. 219.

37 P.E. TAviani, "Si dice che un genovese infedeles cit., p. 8. 
esplorazioni siamo informati grazie ad una deposizione processuale, per il "pleyto» del Darién, resa a San Salvador di Cuba nel febbraio del 1515 dal cognato del medesimo, il pilota Gonzalo Díaz. Il quale, per la spedizione del 1492-93, dichiara: «Bartolomé Garcia (...) vino por contramaestre a esta tierra a descubrir con el dicho Almirante, don Christóval Colón, quando vino la primera vez, el cual se le contó a este testigo e dixo como la primiera tierra, en que dieron, fue Guanahaní e otras islas que dizen de los Yucayos". Per la seconda esplorazione: alla domanda se è notorio che l'Ammiraglio scoprì le molte isole che stanno ad oriente della Hispaniola (Haiti-Santo Domingo), come «San Juan», «Santa Cruz», con tutte le "Yslas de los Canibales», e le "Honze Mill Virgenes", il teste risponde che lo udì affermare da molte persone, in particolare da suo cognato Bartolomé García e dal pilota Christóval Niño, i quali «andovo con el dicho Almirante a descubrir las dichas islas e otras». Per il terzo viaggio: l'inquirente chiede se risulta al teste che la prima scoperta colombiana in "Tierra Firme», cioè nel continente sudamericano, fu Paría (nell'odierno Venezuela), «donde se hallan las perlas», e l'inquisito risponde di sì, "porque lo oyó decir a Bartolomé, Peres Niño e a otras muchas personas que con el dicho Almirante vinieron aquel viaje». Tutte queste informazioni egli ha avuto dall'interessato stesso, circa tredici o quattordici anni prima, cioè tra il 1500 (fine del terzo viaggio dell'Ammiraglio) ed il 1502 (inizio del quarto) ${ }^{38}$.

Risulta altresì che Bartolomé Garcia s'imbarcò infine nella quarta spedizione, come marinaio a bordo della caravella capitana, dietro il compenso di 6.000 maravedís, e morì la domenica 28 maggio 1503, nella rotta delle due navi superstiti - la "Bermuda» e la "Santiago» - tra le isole dei "Jardines de la Reina» e "Cabo Cruz» in Cuba ${ }^{39}$. La presenza di Bartolomé Garcia nell'ultimo viaggio colombiano parrebbe essere di ostacolo ad una sua eventuale identificazione con lo schiavo spagnolo che fornì le informazioni sui tre primi viaggi a Kemâl Reis; ma nulla vieta di supporre che questo marinaio, reduce nel 1500 (od anche prima) dalla terza esplorazione dell' Ammiraglio del Mare Oceano e sempre impegnato sulla flotta reale spagnola, venisse catturato dai Turchi nella battaglia del 1501; andasse libero nel medesimo anno od ai primi del 1502, per riscatto od in altro modo, in tempo per arruolarsi nella nuova piccola flotta del suo antico comandante.

Certo il nome di Bartolomé Garcia non è l'unico che si potrebbe suggerire per la nostra identificazione: resta comunque il fatto che furono pochissimi gli uomini di mare partecipi di tutti i primi tre viaggi colombiani ${ }^{40}$, e che noi non possediamo gli elenchi esatti degli equipaggi di tutte le quattro flotte di Cristoforo Colombo: tre navi nel primo viaggio; diciassette nel secondo (di grande parte delle quali non

38 Escuela de Estudios Hispano-Americanos, Pleitos Colombinos, iII, Probanzas del Almirante de las Indias, Sevilla, 1984, pp. 333-334, 358-359. Bisogna comunque fare attenzione a non confondere lo spagnolo Bartolomé Garcia con il suo omonimo Bartolomé Garcia Genoves, che rese testimonianza a Santo Domingo in Cuba nel giugno 1512, quando aveva venticinque anni o ventisei. Aveva partecipato, quale mozzo a bordo della caravella $\times \mathrm{La}$ Castillaw a circa dieci anni di età, al terzo viaggio di Cristoforo Colombo: cfr. Pleitos colombinos cit., PP. XIX, XX, XXV, XXVI, XXVIII, XXXV, 11, 29; G. PIstarino, Liguri e genovesi nelle flotte di Cristoforo Colombo, in AA.VV., «La storia dei Genovesi», vili, Genova, 1988, pp. 17-32.

39 Crissoforo Colombo, I viaggi dopo la ascopertas cit., p. 347.

40 L. BALletTo, Fra gli equipaggi delle flotte di Cristoforo Colombo, in «Atti del v Convegno internazionale di studi colombiani», Genova, 1990, p. 293. 
conosciamo neppure il nome); otto nel terzo, di cui due nella spedizione del 3 febbraio 1498 al comando di Pietro Fernando Coronel, e sei in quella del successivo 30 maggio, divisesi poi in due parti all'isola del Ferro; quattro nel quarto ${ }^{41}$.

Il Kitab-i Babriye («Libro della marina», "Libro della flotta», "Libro sulla navigazione», "Libro di chi viaggia per mare») raccoglie le annotazioni prese dall'Autore durante i suoi viaggi nel Mediterraneo, con informazioni geografiche, storiche e di carattere navale, allo scopo, come dice lo stesso Piri, di facilitare il lavoro dei naviganti: «Sappi che la lettura di questo libro rende facile l'impegno dei marinai e la capacità d'una persona aumenta come egli apprende tutto circa le scienze importanti. Egli deve leggere ed essere informato sulle varie parti del mondo, e deve anche vederle di per sé. L'uomo saggio è colui che conosce bene se stesso e scopre quante più cose è possibile. Una volta che una persona ha esaurito la propria vita, non può riaverla. Dio sa tutto di noi, e ciò è bene per noi. Perciò Egli può. Egli ci dà tutto ciò che è buono per noi».

Piri mise insieme le sue note nel suo libro del 1521; le rivide e le sviluppò nel 1525 o 1526 (secondo altri studiosi la stesura sarebbe dovuta ad un autore di nome Muradî, ma nulla toglie il merito alla raccolta di Piri Reis). La seconda versione, più raffinata anche nel linguaggio, era destinata al Sultano ed accompagnata da introduzione ed epilogo in versi.

I sette mari del mondo, di cui tratta Piri Reis, sono il Mare Cinese, il Mare Indiano, il Golfo Persico (Bahr-i Pers), il Mare dei Negri (Bahr-i Zenc, intorno a Zanzibar), l'Oceano Atlantico (Bahr-i Magreb o Bahr-i Azam), il Mediterraneo (Bahr-i Rum), il Mare Caspio (Bahr-i Kubzüm), «che è un lago circondato dalla terra».

Piri esamina l'Oceano Atlantico, che egli chiama «Mare Occidentale» o «Grande Oceano». Questo "Mare Occidentale» ha inizio dallo Stretto di Gibilterra e si estende verso occidente per 4.000 miglia. "Ascolta le caratteristiche dell'Oceano Atlantico. E' un mare immenso e tempestoso, dove fino ai tempi recenti nessuno sapeva che cosa fosse. Ora, per volontà divina, vi si può viaggiare. Non credere che sia parte di un mare insulare. Odi e vedi ciò che lo riguarda. Si è tentato a lungo di trovare i suoi confini e di saperne qualcosa, ma nessuno ha trovato dove esso finisce». Piri Reis informa il lettore del continente che egli chiama «Antilia». Nelle sue montagne c'è l'oro, e si trovano perle in fondo al mare, alla profondità di quattro tese. Questo continente fu scoperto dai marinai. Dice testualmente Piri Reis nell'introduzione del libro: "Quella terra si chiama «le Antille». Se siete pronti ad ascoltarmi, vi dirò come quest 'isola fu scoperta. C'era in Genova, un uomo saggio chiamato Kolomp. Gli capitò di trovare un libro interessante, che doveva essere più antico del tempo di Alessandro Magno. Questo libro conteneva ogni informazione sui mari; alla fine esso giunse a questa terra d'Europa. Dapprima non vi si prestò attenzione; ma tosto che il sunnominato Kolomp lo lesse, egli andò dal re di Spagna e gli parlò di ciò che c'era nel libro. Allora il re gli diede delle navi; il saggio uomo

41 P.E. TAviani, I viaggi di Colombo. La grande scopersa, Novara, 1984, ed. pocket, Novara, 1986, pp. 216, $307,392,442$. 
salpò e scoperse quest' isola, aprendo la via a viaggi successivi e tracciando questa rotta famosa. La mappa dell'area è pervenuta in seguito a me; così io sono in grado di parlarvene». Ed ancora: «Egli agì in accordo con questo libro, che rivelò l'esistenza delle Antille. Dopo ciò questo luogo fu aperto al mondo, e la sua rotta fu nota a tutti». Infine: "Al di là ci sono le Antille. Questa terra si trova ai più lontani confini dell'Occidente. In questa terra i giorni e le notti sono di uguale lunghezza. Il sole brilla sempre, ed una metà del mondo è uguale all'altra metà. Voi vorreste sapere se io ho viaggiato colà e se ho visto quelle parti meravigliose. Ad ogni modo sappiate che la temperatura è di quarantasette centigradi».

Piri Reis è famoso anche in ambienti non specialistici, dato che in tempi recenti persino l' "ufologia» e la fantascienza se ne sono impadronite ${ }^{42}$.

Su Genova, che Piri Reis dichiara patria di Cristoforo Colombo -e la sua testimonianza è fondamentale-, il nostro Autore si mostra bene informato. Nel Kitab-i Babriye ci dà un'esatta descrizione del porto; come pure conosce, e vi accenna per sommi capi, le vicende della città, che tra il 1499 ed il 1528 subì ripetute dominazioni francesi, mentre nel maggio del 1522, proprio quando Piri attendeva alla sua opera, sofferse l'occupazione ed il terribile saccheggio delle truppe spagnole, comandate da Prospero Colonna e dal marchese di Pescara. Ritornata sotto la dominazione francese nel 1527, Genova passò in protezione della Spagna nel 1528 con Andrea Doria. Riemerge di qui la conferma di come nel mondo ottomano si conoscessero perfettamente e si seguissero con attenzione le vicende dell'Occidente cristiano. Ma lasciamo la parola al nostro Autore, che prende le mosse dal territorio del Dominio:

"Questa regione era prima governata dai mercanti di Genova (Ceneviz). Poi la conquistò il re di Francia; poi la Spagna la occupò, togliendola al re di Francia. Ora Genova è sottoposta al re di Spagna. E' una grande città sulla riva del mare verso sud, costruita sul pendio della montagna. Dietro la città sono tutte montagne. Davanti alla città sono stati costruiti un porto ed un mandracchio. Si entra dalla parte sud nel porto, poi si vira verso est e si gettano le ancore. Ma è un porto stretto. Le navi che entrano si ancorano legandosi sia a poppa sia a prua. Le navi piccole entrano fino in fondo alla parte orientale e vi si ancorano. Le grandi caracche, cioè le göke molto grosse, si ancorano sotto la torre che sta dalla parte orientale all'imboccatura del porto. Infatti dalle due parti della bocca del porto ci sono due alte torri, nelle quali ogni sera si accendono i fari: sono per fare entrare nel porto con facilità le navi dei mercanti che passano di notte. Entrate nel porto, si avvicinano alla torre che sta dalla parte orientale. Ma le grandi caracche, quando sono entrate nel porto, girano dalla parte est della torre orientale, entrano e, legandosi saldamente a poppa e a prua, si ancorano verso nord-ovest. Davanti all'alta torre, che sta ad ovest, fuori del porto, c'è un golfo, che in effetti è un porto, dove si costruiscono le navi» ${ }^{43}$.

Uscendo da Genova, lungo la Riviera di Levante, è indicato con sufficiente

42 Rappresentante di questa tendenza è il libro di L. Ouvieri, L'Aslantide ba lasciato una traccia, Roma 1979. ma la letteratura sull'argomento è piuttosto vasta. Ancora più dettagliato C.H. HAPGOOD, Maps of the Ancient Sea Kings, Philadelphia-New York, 1966.

27.

43 A. BAUSANI cit., p. 194, riprodotto in G. M ARCENARO, Viaggiatori stranieri in Liguria, Genova, 1987, p. 
precisione un tratto del percorso della Lunigiana meridionale fino alla Toscana. A cinque miglia da Qavo Qorbo (Capo Corvo) c'è Fose de le Magra (Foce di Magra), cioè "l'insenatura della Magra», dove entrano le piccole navi: essendo l'acqua alquanto bassa, le grandi navi non vi possono entrare. Di qui a Ele-Vinse (l'Avenza) sono tre miglia; da Ele-Vinse a Monteron (Motrone), sette; da Motrone a Fose d'Arno (foce dell'Arno), dieci ${ }^{44}$.

Non possediamo gli originali del Babriye: né dell'edizione del 1521 né di quella del 1525 o 1526. Manoscritti della prima si trovano ad Istanbul, a Bologna, a Vienna, a Dresda, a Berlino, a Parigi, a Londra; della seconda, numerosi, ad Istanbul. E' la fortuna di un "masterpiece in the science of navigation" ".

La parte relativa alla Sicilia è stata pubblicata, con traduzione in tedesco, da E. Sachau, Sicilien nach dem türkischen Geographen Piri Reis, in "Centenario della nascita di Michele Amari», II, Palermo, 1910.

Come già si è ricordato, un' edizione parziale, condotta con metodo scientifico e con traduzione tedesca, è stata pubblicata da P. Kahle, Piri Reis. Baberiye, nel 1926, sulla base della prima stesura dell'opera e solo fino a Rodi inclusa. Della seconda versione esiste un'edizione in facsimile sulla scorta del ms. Aya Sofya, 1612, a cura della Fondazione per le Ricerche storiche turche, sotto il titolo: Piri Reis, Kitab-i Babriye, Istanbul, 1935. Un'edizione dell'opera intera secondo il ms. Aya Sofya 2612 , in turco modernizzato, ma con errori nella trascrizione e identificazione dei toponimi, è stata condotta da Yavuz Senemog̃lu, Piri Reis, Kitab-i Babriyye, voll. 2, s.l., 1973. Servendosi di questa, ma con il confronto con il testo originale ottomano, Alessandro Bausani ha compiuto la traduzione italiana degli excerpta della parte italiana (escluse la Sardegna e la Sicilia), nel saggio, già qui citato, L'Italia nel Kitab-i Babriyye di Piri Reis, in "Il Veltro. Rivista della civiltà italiana», XXIII, num. 2-4, marzo-agosto 1979, Le relazioni tra l'Italia e la Turchia, pp. 173-196.

Come scrive A. Afetinan: «Piri Reis lived in an age in which Turkish culture was flourishing in almost every field. The XVth century can be looked upon as the golden age of Turkish civilisation. In such an age as this, Piri Reis contributed to the science of the world by means of his works, and appeared as a figure who must be given a high place in the history of civilisation» ${ }^{46}$. E Taviani: "La mappa di Piri Reis e la sua opera Babriye sono prove eloquenti dell'alto livello raggiunto dalla civiltà turca»" ${ }^{47}$.

\author{
Geo Pistarino \\ Accademia Lignre di Scienze e Lettere \\ (Genova, Italia)
}

\footnotetext{
44 A. BAUSANI cit., p. 195.

45 A. Afetinan, Life and Works cit., p. 59.

46 A. APETINAN, Life and Works cit., p. 50.

47 P.E. Taviani, "Si dice che un genovese infedele» cit., p. 8.
} 